\title{
ANALYSE CHIMIQUE DES SOLIDES NON CRISTALLINS EN SURFACE ET EN COUCHES MINCES
}

\author{
J. TOUSSET
}

Institut de Physique Nucléaire (et IN2 P3), Université Claude-Bernard Lyon-1, 43, Bd du 11-Novembre 1918, 69621 Villeurbanne, France

\begin{abstract}
Résumé. - L'analyse chimique des solides non cristallins en surface et en couches minces se révèle de plus en plus nécessaire et pose cependant souvent un difficile problème d'effet de charge pour beaucoup de méthodes. Sans naturellement être exhaustive, une courte description des principales méthodes (spectrométries de masse, méthodes nucléaires et spectroscopies électroniques) est exposée.
\end{abstract}

\begin{abstract}
The surface analysis of major and minor elements in non-crystalline solids presents a challenging problem. Many surface analysis techniques, which involve the caracterization of surfaces and the determination of in-depth concentration profiles are hampered by a charging effect in these materials. Among the possible analytical methods, we have chosen the mass spectrometry techniques, nuclear methods and electron spectroscopies (ESCA in fact).
\end{abstract}

Introduction. - L'analyse des matériaux non cristallins se heurte très souvent à deux problèmes : la résistance chimique pour les analyses par voie humide et le caractère isolant pour les méthodes physiques de caractérisation. Parmi l'ensemble des méthodes disponibles, nous n'envisagerons pas ici les méthodes d'analyse dans la masse (par voie humide ou non): absorption atomique, spectrophotométrie d'émission, fluorescence $X$, spectroscopies IR, etc... et nous nous limiterons à quelques méthodes d'analyse de couches minces et de surface.

L'importance de ce domaine est grandissante. On sait en effet que la surface n'a pas toujours la même composition que la masse par suite de vaporisation, de migrations de surface, ... durant la fabrication, ou contaminations et corrosions ultérieures, etc...; et cependant cette composition affecte directement de nombreuses propriétés du produit préparé. Par ailleurs, de nombreuses couches minces amorphes sont actuellement produites et étudiées : leur caractérisation (gradient de composition, impuretés...) est primordiale.

Le tableau I non exhaustif, situe quelques méthodes par rapport à leurs possibilités moyennes d'analyse en profondeur.

Notre objectif sera ici un examen sommaire des plus importantes d'entre elles en les groupant en trois classes.

1. Les méthodes basées sur l'analyse des ions produits, c'est-à-dire la spectrométrie de masse à étincelles, la sonde à laser, l'analyseur ionique.

2. La microanalyse nucléaire, c'est-à-dire essentiellement l'observation directe de réactions nucléaires et de la rétrodiffusion coulombienne.

3. Les spectroscopies électroniques Auger et surtout ESCA.

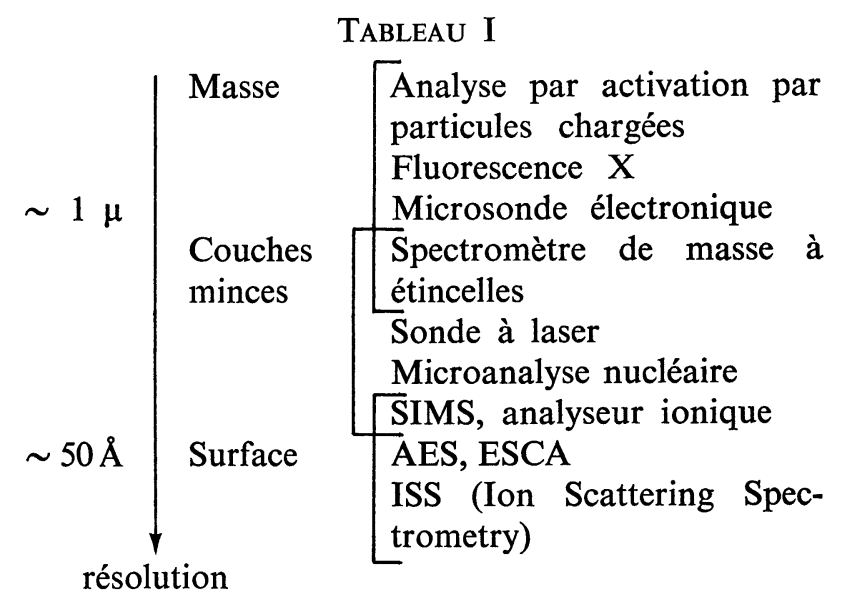

1. Les spectrométries de masse. - a) On sait que la spectrométrie de masse à étincelles permet d'obtenir un panorama de composition avec une bonne sensibilité et qu'elle peut fournir directement l'analyse semi-quantitative. Bien que ce ne soit pas à proprement parler une méthode d'analyse de surface, on peut obtenir l'étincelage [1] à l'aide de deux électrodes auxiliaires disposées très près de l'échantillon, permettant la formation d'un plasma sur la surface du matériau isolant à analyser. Le procédé s'applique par exemple à l'étincelage d'une fibre optique.

Quant aux résultats, chaque type de verre manifeste un comportement particulier, du fait de la présence d'oxygène, de sodium, de silicium qui s'ionisent bien, les associations atomiques du type $\mathrm{X}_{\mathrm{n}} \mathrm{O}_{\mathrm{m}}^{+}, \mathrm{X}_{\mathrm{n}} \mathrm{O}_{\mathrm{m}} \mathrm{Y}_{\mathrm{p}}^{+}$ sont très nombreuses et le pouvoir de résolution du spectromètre doit dépasser 4000 vers les masses 60 .

Beaucoup d'éléments sont décelés à partir de teneurs aussi basses que $15 \mathrm{ng} / \mathrm{g}[1,2,3]$.

b) L'impact d'un faisceau laser à rubis type 
déclenché $(\lambda=6943 \AA)$ correspondant à une densité de puissance élevée (entre $10^{8}$ et $10^{9} \mathrm{~W} . \mathrm{cm}^{-2}$ ) provoque une brutale élévation de la température $\left(10^{4} \mathrm{~K}\right)$ dans un petit volume $\left(10^{-4}\right.$ à $\left.10^{-7} \mathrm{~mm}^{3}\right)$, ce qui conduit à la volatilisation, atomisation et ionisation des constituants. L'état physique de cette matière surchauffée ressemble à celui d'un plasma où toutes les liaisons chimiques sont rompues et les atomes dans un état d'ionisation presque totale $[4,5,6]$.

L'analyse des ions positifs est réalisée magnétiquement après une accélération de $12 \mathrm{kV}$. Le diamètre de l'impact est compris entre 15 et $200 \mu$ pour une profondeur de 0,1 à $5 \mu$. Cette spectrométrie de masse permet ainsi l'examen d'échantillons isolants sans préparation spéciale ; elle possède de plus une meilleure résolution spatiale que la spectrométrie à étincelles et fournit un spectre de masse simple où les ions multichargés sont rares et les ions moléculaires négligeables. On peut, de plus, nettoyer la surface avant l'analyse par un tir laser initial de faible énergie. On peut aussi multiplier les tirs pour tracer un profil de concentration jusqu'à $100 \mu$. Le seuil de détection des éléments est de quelques $10^{-6}$.

c) Par ailleurs, on sait que le bombardement par des ions argon (ou autres) provoque l'émission d'ions secondaires qui, analysés, peuvent conduire au dosage des éléments constituants, au tracé de profils de concentration, ou (et) à l'obtention d'une image.

Cette technique d'analyse d'ions secondaires n'est pas applicable directement aux matériaux isolants qui se chargent électriquement $[7,8,9]$ principalement sous l'effet du bombardement primaire. Pour pallier cet inconvénient, on peut :

- soit compenser la tension induite par les charges en modifiant la tension du porte-échantillon,

- soit neutraliser ou diminuer les charges positives de surface.

Dans le premier cas $[10,11]$, on doit compenser le supplément d'énergie des ions secondaires en décalant la tension de l'objet grâce à un potentiel ajustable. Cette tension de correction peut atteindre 200 volts sur des couches de silice de $8000 \AA$. L'inconvénient principal de ce procédé est de ne pas empêcher les migrations éventuelles d'impuretés sous l'effet du champ électrique $[12,5]$. Ce procédé ne semble pas non plus applicable de façon systématique.

Dans le second cas, plusieurs solutions ont été proposées :

- dépôt par évaporation d'une grille métallique sur la surface de l'échantillon. Cette grille est gênante pour le tracé de profils ;

- bombardement avec des particules neutres, mais le résultat n'est pas évident. Des améliorations intéressantes sont en cours ;

- dépôt d'un diaphragme métallique sur l'échantillon avec emploi d'un faisceau d'ions défocalisé. La production électronique de ce métal semble suf- fisante pour neutraliser la charge se produisant sur la faible surface de l'isolant bombardé ;

- bombardement électronique. Le résultat paraît satisfaisant.

Moyennant donc des précautions adéquates, l'analyseur ionique est un outil particulièrement précieux pour l'analyse de solides isolants (par exemple [13]). La résolution en profondeur est de l'ordre de 50 à $100 \AA ̊$.

2. La microanalyse nucléaire. - Les méthodes nucléaires sont en fait assez variées et on peut les séparer schématiquement en trois classes :

- Utilisation des traceurs radioactifs, soit pour jouer le rôle d'indicateur dans des processus de diffusion, etc..., soit à la base de méthodes comme les dilutions isotopiques, etc... Cette utilisation ne sera pas envisagée ici.

- Analyse par activation, c'est-à-dire production grâce à un bombardement de particules appropriées (souvent neutrons) de radio-éléments dont la détection différée permet de remonter à la composition. Cette méthode connue, très sensible, est surtout utilisée pour l'analyse dans la masse.

- Microanalyse nucléaire, c'est-à-dire observation directe des réactions nucléaires ou des désexcitations atomiques.

Ces méthodes nécessitent généralement l'usage d'un petit accélérateur du type Van de Graaff $2 \mathrm{MeV}$.

La production de RX sous faisceau d'accélérateur est à la base d'une méthode qui a connu ces dernières années un développement certain. Les grandes sections efficaces d'excitation, le faible bruit de fond assurent à l'analyse une grande sensibilité et la rapidité. Le faible parcours des ions incidents $(p, \alpha$, ions lourds) et l'absorption du rayonnement émis en font une méthode d'analyse superficielle spécialement adaptée à l'analyse de couches minces complexes. Le dosage simultané de nombreux éléments est ainsi possible, sans cependant de résolution en profondeur appréciable (application: vernis sur céramique ancienne, par exemple).

L'observation directe de réactions nucléaires proprement dites $(d, p),(p, \alpha)$, etc... ou de la simple rétrodiffusion élastique s'est révélée un outil puissant de caractérisation de zones superficielles ou de couches minces (par exemple [14]), ses caractéristiques dominantes peuvent se résumer ainsi :

- la méthode est non destructive,

- vu les énergies des particules détectées, les faibles courants, les parcours et les processus d'interaction, le caractère éventuellement diélectrique de la couche analysée est sans incidence notable,

- l'analyse est indépendante de l'état chimique,

- la profondeur analysée peut dépasser quelques microns, 
- la résolution en profondeur atteint moins de $100 \AA$ en surface, dans les cas favorables,

- la résolution latérale est faible (généralement de l'ordre du $\mathrm{mm}^{2}$ ),

- l'analyse peut facilement être quantitative (5\% ou mieux), sensible et assez rapide.

Le caractère non destructif, la possibilité d'analyse sur plusieurs milliers d'angströms avec une bonne résolution moyenne en font une excellente méthode de caractérisation de couches minces.

L'utilisation de réactions nucléaires ou de la simple rétrodiffusion fournit deux moyens complémentaires pour résoudre un ensemble de problèmes :

a) L'observation de réactions nucléaires convient bien à l'étude d'éléments légers sur lesquels ces réactions peuvent facilement se produire malgré la faible énergie des particules incidentes. Leur bilan énergétique est de plus, souvent positif, ce qui permet aux particules émises de se différencier aisément du fond intense des particules simplement rétrodiffusées.

Un exemple se trouve dans les réactions $(d, p)$ sur l'oxygène et le silicium [15] pour l'étude de la stœechiométrie de couches minces de silice amorphe (Fig. 1).

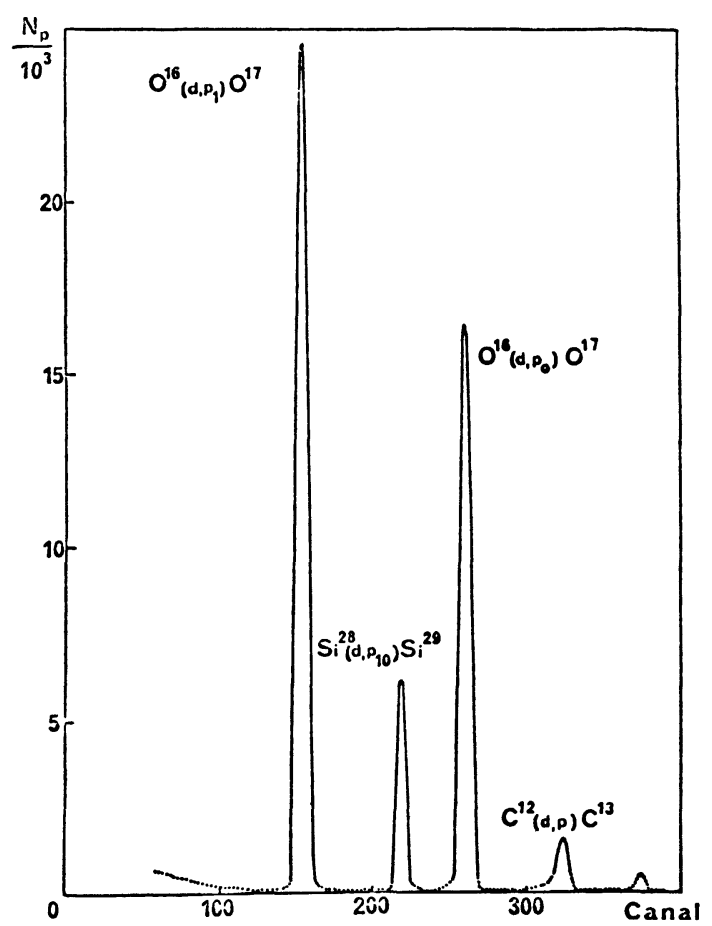

Fig. 1. - Spectre énergétique de protons issus d'une couche d'oxyde de silicium de $4000 \AA$. $E_{\mathrm{d}}=1,5 \mathrm{MeV}$ [15].

La présence de pics voisins dus respectivement au silicium et à l'oxygène permet une détermination assez précise et sûre du rapport stœechiométrique (meilleure que $1 \%$ ). La couche est assez mince (4 $000 \AA \AA)$ pour que les variations de sections efficaces par suite de la perte d'énergie soient négligeables.

Un autre exemple peut être fourni par une étude REVUE de PHYSIQUe APPLIQUÉE. - T. 12, No 5, MAI 1977 du profil de sodium à la surface de verre par la réaction ${ }^{23} \mathrm{Na}(\mathrm{p}, \alpha){ }^{20} \mathrm{Ne}$ [16]. Les auteurs ont pu montrer que l'analyse est possible dans des conditions où la diffusion du sodium sous la mesure n'est pas à craindre. Dans ce cas, il s'agit d'une réaction à résonance que l'on enfonce progressivement dans l'échantillon en augmentant lentement l'énergie incidente. C'est encore le cas de la réaction ${ }^{32} \mathrm{~S}\left(\mathrm{p}, \mathrm{p}^{\prime} \gamma\right){ }^{32} \mathrm{~S}$ qui permet [17] de tracer le profil de répartition du soufre dans une couche de 1,7 $\mu$ d'un verre AsGeTeS, obtenue par évaporation-flash. La répartition du lithium à la surface de verre a été étudiée par la réaction ${ }^{7} \mathrm{Li}(\mathrm{p}, \gamma){ }^{8} \mathrm{Be}[18]$.

b) La détection de particules simplement rétrodiffusées complète bien l'observation de vraies réactions nucléaires. Elle présente en plus l'avantage d'une grande simplicité d'interprétation (ne pas confondre cette rétrodiffusion à haute énergie avec sa sœur ISS (Ion Scattering Spectrometry) à très basse énergie ( $\sim$ quelques $\mathrm{keV}$ ou moins)).

La résolution en profondeur, liée en particulier à la perte d'énergie par unité d'épaisseur, peut être optimalisée par un choix judicieux de la particule incidente (par exemple ions $\mathrm{Li}^{+}$au lieu de $\mathrm{p}$ ou $\alpha$ [19]) ou par l'artifice d'opérer à petits angles par rapport à la surface [20].

Cette méthode est donc fort utile pour les analyses de couches minces d'oxydes anodiques par exemple [21]. La figure 2 [22] représente le contrôle
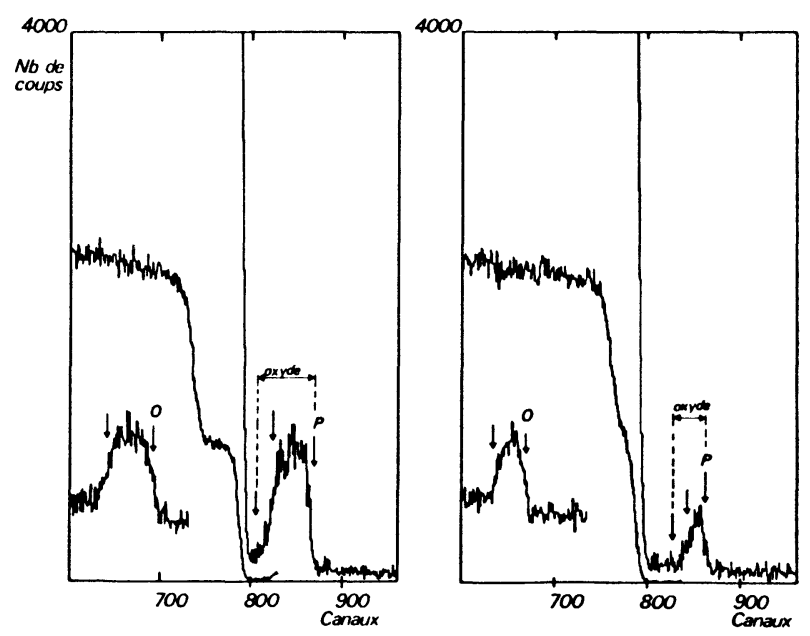

Fig. 2. - Spectre énergétique de particules $\alpha$ rétrodiffusées $\left(E_{0}=2,2 \mathrm{MeV}\right)$ montrant la répartition du phosphore dans une couche d'oxyde d'aluminium anodique formée à $60 \mathrm{~V}$ : a) avant abrasion, $b$ ) après abrasion [22].

d'une couche de $900 \AA$ environ d'alumine formée en bain phosphorique dont on peut vérifier la stœchiométrie (à 3-5\%) et la répartition du phosphore. On trouve que le phosphore est réparti de façon homogène dans la couche à une teneur de l'ordre de $5 \%$ par rapport à l'aluminium et sur environ les $2 / 3$ de la couche (flèches extrêmes, Fig. a). Une abrasion ionique permet une meilleure appréciation de la 


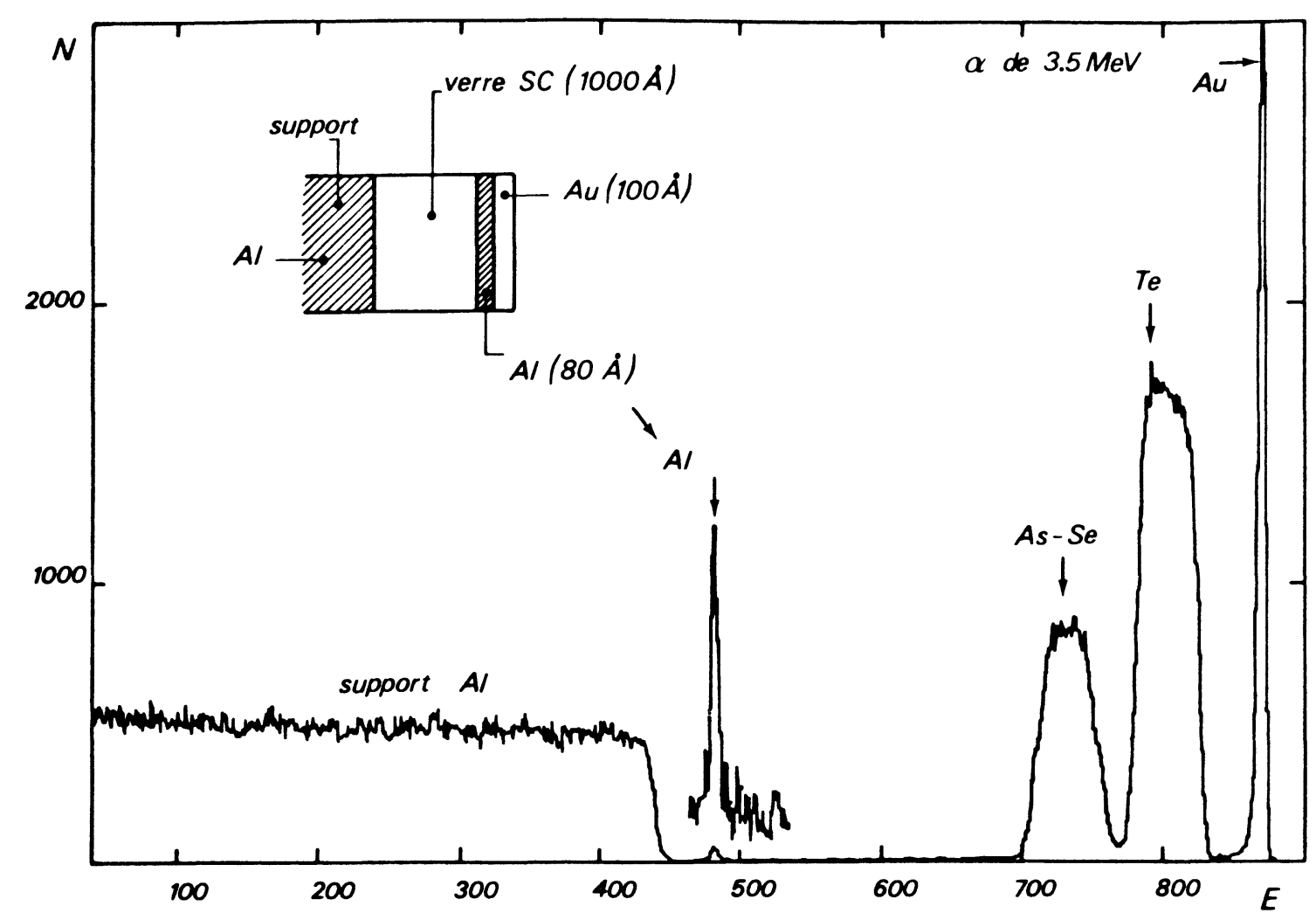

FIG. 3. - Spectre énergétique de particules $\alpha$ rétrodiffusées sur un dispositif à base de verre As-Te-Te $\left(E_{0}=3,5 \mathrm{MeV}\right)$.

répartition du phosphore dans la dernière portion de la couche, la largeur du pic oxygène précisant l'épaisseur (Fig. $b$ ).

Il est de même possible de situer la position et l'importance d'espèces implantées (par exemple [23]), d'étudier des diffusions thermiques ou des électromigrations, au moins dans les cas favorables. La figure 3 représente par exemple le spectre de rétrodiffusion de particules $\alpha$ de $3,5 \mathrm{MeV}$ sur un dispositif semiconducteur amorphe As-Se-Te déposé sur aluminium et recouvert d'une électrode complexe or-aluminium. L'évolution du spectre en cours de traitements thermiques permet de suivre la diffusion de l'électrode, le rôle de la couche barrière, etc...

3. Les spectroscopies électroniques ESCA et AUGER. - Elles correspondent à des études de zones beaucoup plus superficielles (quelques dizaines d'angströms) et de ce fait se révèlent souvent complémentaires des méthodes nucléaires dont le domaine privilégié est celui des couches minces multiples (quelques centaines à quelques milliers d'angströms).

Nous limiterons arbitrairement notre examen à la spectroscopie ESCA, en signalant que la spectroscopie Auger fréquemment utilisée (cf. par exemple [46]) rencontre souvent des problèmes délicats d'effets de charge provoquant des migrations, etc... [25]. Cette spectroscopie Auger présente l'avantage d'une analyse localisée, mais des effets de bombardement généralement plus sérieux qu'en ESCA.
La spectroscopie ESCA (Electron Spectroscopy for Chemical Analysis) repose sur l'effet photoélectrique. Elle consiste en la mesure précise de l'énergie de photoélectrons émis par un échantillon bombardé avec un rayonnement $X$ monochromatique. La conservation de l'énergie permet d'en déduire avec précision l'énergie de liaison des électrons. Ces énergies de liaison électronique sont caractéristiques de la nature des atomes constituants. Leur détermination permet l'identification des atomes, donc l'analyse élémentaire de n'importe quel atome possédant des électrons de cœur (tous les éléments, sauf $\mathrm{H}$ ).

De plus, ces énergies de liaison des électrons de cœur peuvent subir de légères modifications suivant l'environnement chimique de l'atome. La mesure de ces déplacements est très importante pour obtenir une information chimique précieuse. Par ailleurs, le tracé précis de la bande de valence laisse prévoir des renseignements directs sur les liaisons chimiques. Eventuellement, l'existence de pics satellites (shake-up, plasmons, ...) fournit des indications complémentaires.

Sur le plan pratique, l'échantillon est bombardé sur une surface de l'ordre $\mathrm{du} \mathrm{cm}^{2}$ ou plus petite. Une caractéristique importante concerne la profondeur analysée. Les électrons qui portent l'information intéressante sont ceux qui n'ont subi aucune interaction après leur émission. Ils sont donc issus de couches très superficielles. La profondeur d'analyse dépend donc du libre-parcours moyen de ces électrons (l'atténuation des RX est négligeable devant ces profondeurs). Ces 
libres-parcours moyens, fonction de l'énergie cinétique des électrons varient de 5 à $50 \AA$ entre 50 et $2000 \mathrm{eV}$. Ils dépendent aussi du matériau, mais moins nettement. Cela nous conduit à dire que l'analyse s'effectue sur une épaisseur de l'ordre d'une cinquantaine d' $\AA$ ou moins.

Une difficulté attendue dans le cas de composés isolants est évidemment l'effet de charge qui provoque une dérive des pics en modifiant l'énergie des électrons et un élargissement néfaste. Bien sûr, tout effet de charge peut aussi créer des migrations parasites d'éléments recherchés. Cet effet de charge a suscité beaucoup de travaux et, là aussi, on rencontre les deux solutions possibles:

- en tenir compte par un étalonnage adéquat,

- essayer de le supprimer.

Pour la première solution, on peut par exemple déposer un mince dépôt d'or par évaporation sur l'échantillon [26]. On sait que l'or se met alors en petits îlots isolés prenant le potentiel de la surface isolante étudiée. Les pics photoélectroniques de l'or permettent alors un calibrage correct. On s'est aussi servi du dépôt de carbone issu de l'huile de pompage dans les installations à vide non propres. Pour la seconde solution, il est possible d'envisager l'usage d'un canon à électrons ou encore d'utiliser un portecible métallique en forme de cuvette en comptant sur les photoélectrons issus des bords de ce porte-cible également bombardé par les RX incidents pour neutraliser la charge positive apportée par le départ des photoélectrons de l'échantillon isolant [27]. Dans ce dernier cas, un verre de silice de $1 \mathrm{~mm}$ d'épaisseur produit les pics du doublet $\mathrm{Si} 2 \mathrm{p}$ à une valeur correcte de l'énergie; l'effet de charge est annulé.

$\mathrm{Vu}$ probablement les difficultés précédentes, ou l'acuité de problèmes de surface posés avec les métaux ou aussi l'intérêt théorique de travailler avec des monocristaux, le nombre de travaux consacrés aux matériaux non cristallins est moins fourni jusqu'ici. On peut voir se dessiner trois types d'études :

- l'analyse élémentaire des surfaces dont la composition est supposée différente de la masse

- l'analyse de l'ordre local en se basant sur les déplacements chimiques des électrons de cœur

- l'analyse structurale également, grâce à l'examen attentif de la bande de valence.

3.1 ANALYSE ÉlÉMENTAIRE DES SURFACES. - La figure 4 [28] représente un spectre ESCA typique. Il s'agit d'un verre au phosphate de calcium contenant du fer. Les deux spectres illustrent l'aspect de surface de l'examen ESCA.

La possibilité de réaliser une analyse quantitative ou au moins semi-quantitative sur des verres est démontrée par plusieurs travaux $[20,30,31,32,33$, $34,35,36,37]$. Un effort important a été porté sur les enrichissements ou appauvrissements superficiels d'éléments tels que le sodium, le calcium, etc... C'est ainsi

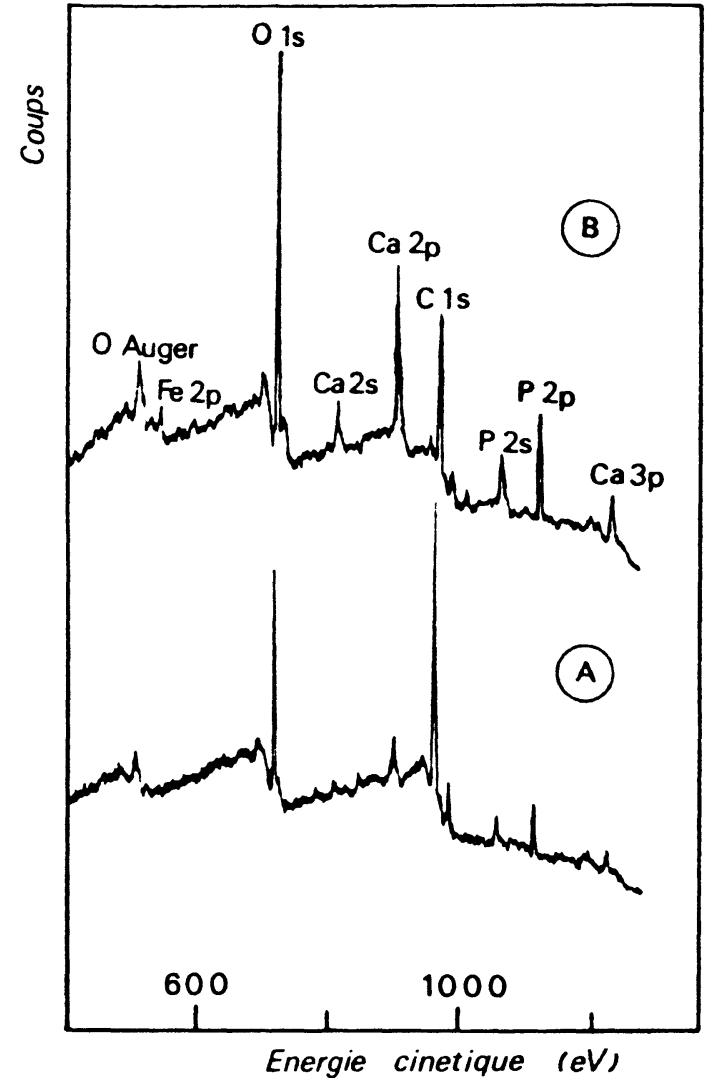

Fig. 4. - Spectre ESCA d'un échantillon de verre au phosphate de calcium : a) Avant décapage à l'argon. b) Après ce décapage [28].

que les fibres de verre $\mathrm{A}$ montrent généralement un appauvrissement très net $(50 \%)$ de la teneur superficielle en calcium, indépendamment du vieillissement [30], mais cette teneur augmente avec la température [29].

L'évolution de la teneur superficielle en sodium en fonction du temps d'exposition à l'eau de verres variés est remarquable. C'est ainsi que pour un verre industriel [32] de composition $\mathrm{SiO}_{2} 73,9 \%, \mathrm{Na}_{2} \mathrm{O}$ $16 \%, \mathrm{CaO} 7,4 \%$, la concentration en sodium s'effondre rapidement (d'un facteur voisin de 7) dans les 30 premiers angströms et décroît ensuite lentement avec le temps. Après 5 à 6 mois d'exposition à l'eau, le sodium a quasi disparu de la surface. Pour un autre verre expérimental $\left(\mathrm{SiO}_{2} 69,7 \%, \mathrm{Na}_{2} \mathrm{O}\right.$ $30,3 \%$, la concentration superficielle en sodium passe par un minimum et semble ensuite tendre vers sa valeur initiale. C'est le comportement d'une couche superficielle d'un verre non résistant à l'eau.

On note que l'utilisation du pic Na1s de plus forte énergie de liaison $(1072 \mathrm{eV})$ donc de plus basse énergie cinétique $(182 \mathrm{eV})$ accentue le caractère superficiel de l'analyse par rapport aux pics $\mathrm{Na} 2 \mathrm{~s}(63 \mathrm{eV})$ (donc $\left.E_{\text {cin }}=1191 \mathrm{eV}\right)$ ou Auger Na KLL $\left(E_{\text {cin }}=988 \mathrm{eV}\right)$.

3.2 ANALYSe De l'État ChimiQue. - L'analyse de l'état chimique peut se réaliser grâce aux déplacements 
en énergie des électrons de cœur, qui traduisent des sites chimiques différents.

C'est ainsi que pour un verre $\mathrm{Pd}-\mathrm{Si}\left(\mathrm{Pd}_{\mathbf{8 4}} \mathrm{Si}_{16}\right)$ l'enregistrement de spectres ESCA successifs entrecoupés d'abrasions ioniques permet de mettre en évidence que le silicium en surface est essentiellement sous forme d'oxyde [38].

Un autre exemple (Fig. 5) [39] concerne la composition de polymères fluorés. Les pics $\mathrm{C} 1 \mathrm{~s}$ de cœur correspondent à des atomes de carbone dans des environnements différents, pour deux polymères.

De même, pour des verres $\mathrm{As}_{2} \mathrm{Se}_{3}$ et $\mathrm{As}_{4} \mathrm{Se}_{4}$ [40],

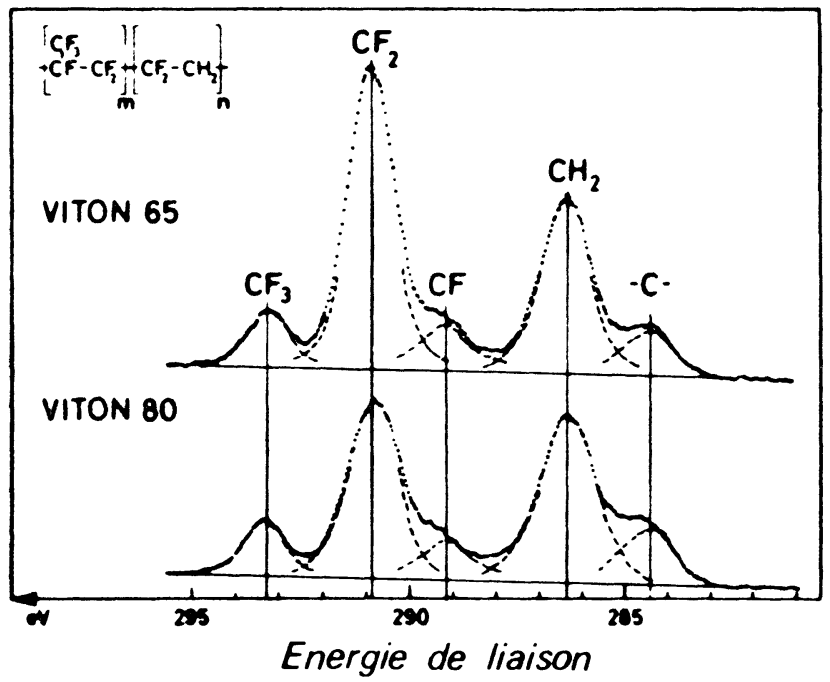

FIG. 5. - Spectre ESCA de polymères-carbone 1s [39]. les déplacements par rapport aux éléments As et $\mathrm{Se}$ sont identiques pour le sélénium mais différents pour l'arsenic dans les deux verres, ce qui tend à montrer que les liaisons locales du sélénium sont les mêmes dans les deux verres, mais différentes pour l'arsenic (cf. aussi [41, 42]).

\subsection{ANALYSE STRUCTURALE GRÂCE AUX ÉLECTRONS} DE VALENCE. - L'ESCA utilisée avec un rayonnement $\mathrm{X}$ incident monochromatique fournit une excellente description de la bande de valence. C'est de ce fait un outil potentiel exceptionnel pour les études des liaisons chimiques. Il est souvent dans ce cas couplé avec les études en UPS, c'est-à-dire la spectroscopie électronique sœur, utilisant un rayonnement UV. Malheureusement l'interprétation des différentes structures de cette bande est délicate et doit se faire en corrélation avec des calculs théoriques difficiles d'orbitales moléculaires ou de densités d'états électroniques. Des travaux déjà nombreux ont été entrepris dans l'espoir de montrer une différence entre état cristallin et amorphe, conduisant à une interprétation liée à la structure.

Dans le cas par exemple du sélénium [43, 44, 45], plusieurs interprétations sont proposées pour expliquer les modifications observées entre les densités d'état à l'état cristallisé ou à l'état amorphe [45] : augmentation de la distance interchaine [44], diminution de l'angle dihédral de $102^{\circ}$ (Se trigonal) à environ $80^{\circ}$ [43], ou signe de l'angle dihédral alterné ou au hazard [45]. Ce genre d'étude n'en est qu'à ses débuts.

\section{Bibliographie}

[1] Gauneau, M., Analysis 3 (1975) 368.

[2] German, B., Scaplehorn, A. W., J. Forensic Sci. Soc. 12 (1972) 367.

[3] Stefani, R., 7th Intern. Mass Spectrometry Conference, Florence, Sept. 1976.

[4] Bingham, R. A., Salter, P. L., Anal. Chem. 48 (1976) 1735.

[5] Eloy, J. F., Stefani, R., Cornu, A., Bouchez, R., DesHAYES, J. J., IX ${ }^{\mathrm{e}}$ Congrès de l'Union Internationale des Sciences Préhistoriques et protohistoriques, Nice, Sept. 1976.

[6] Eloy, J. F., Stefani, R., XVIII ${ }^{\mathrm{e}}$ Colloquium Spectroscopicum Intern,. GAMS, Grenoble, Sept. 1975.

[7] Slodzian, G., Dennebouy, R., Havette, A., XVIII ${ }^{\mathrm{e}}$ Colloquium Spectropicum Intern., GAMS, Grenoble, 1975.

[8] Werner, H. W., Morgan, A. E., J. Appl. Phys. 47 (1976) 1232.

[9] Marguerite, J. L., Thèse, Grenoble, Nov. 1976.

[10] Hilleret, N., Thèse Grenoble, 1973.

[11] Blanchard, B., Carrier, P., Hilleret, N., Marguerite, J. L., Rocco, J. C., XVIII ${ }^{\mathrm{e}}$ Colloquium Spectroscopicum Intern., GAMS, Grenoble, Sept. 1975.

[12] McCaughan, D. V., Kushner, R. A., Characterization of Solid Surfaces (Ed. P. F. Kane et G. R. Larrabee), (Plenum Press, New York) 1974.

[13] Scherrer, S., Naudin, F., Communication personnelle, $\mathrm{XI}^{\mathrm{e}}$ Congrès Intern. du Verre, Prague 1977 (à paraître)

[14] Chu, W. K., MaYer, J. W., Nicolet, M. A., BucK, T. M., AMsel, G., Eisen, F., Thin Solid Films 17 (1973) 1.

[15] Cachard, A., Pivot, J., Roger, A., Talvat, M., Thomas, J. P., Revue Phys. Appl. 6 (1971) 279.
[16] Carnere, A., Della Mea, G., Drigo, A. V., Lo Russo, S., Mazzoldi, P., Rapport interne, Instituto di Fiscica dell' Universita, Padova.

[17] Porte, L., Thèse Doct. Spéc. $3^{\mathrm{e}}$ cycle, Lyon, 1972.

[18] Engelmann, Ch., Golicheff, I., Loeuillet, M., Silic. Ind. 3 (1974) 59.

[19] Thomas, J. P., Cachard, A., Fallavier, M., Tardy, J., Marsaud, S., Pivot, J., Revue Phys. Appl. 11 (1976) 65.

[20] Williams, J. S., "Ion Beam Surface Layer Analysis », vol. 1, 223 (ed. O. Meyer, G. Linker, F. Kappeler) (Plenum Press, New York) 1976.

[21] Thomas, J. P., Cachard, A., Fallavier, M., Tardy, J., MARSAUd, S., "Ion Beam Surface Layer Analysis", vol. 1, 425 (ed. P. Meyer, G. Linker, F. Kappeler), (Plenum Press, New York) 1976.

[22] Fallavier, M., Thèse Doct. Spèc. $3^{\mathrm{e}}$ cycle, Lyon, (1977).

[23] Benmalek, M., Thomas, J. P., Mackowski, J. M., Intern. Conf. on Ion Implantation in Semiconductors and other materials, Boulder, Colorado, Août 1976.

[24] Marsaud, S., Thèse Doct. Spéc. $3^{\mathrm{e}}$ cycle, Lyon (1976).

[25] Pantano, C. G., Jr, Dove, D. B., Onoda, G. Y., Jr., J. Vac. Sci. Technol. 13 (1976) 414.

[26] Ginnard, C. R., Riggs, W. M., Anal. Chem. 46 (1974) 1306.

[27] Feve, L., Fontaine, R., Caillat, R., Cassir, M., $C$. $R$. Hebd. Sèan. Acad. Sci. 282 (1976) C-1029.

[28] Tricker, M. J., Winterbottom, A. P., Bishay, A., MouTRAN, R. F., J. Non-Crystal. Solids 16 (1974) 303.

[29] Nichols, G. D., Hercules, D. M., Peak, R. C., VauGHAN, D. J., Appl. Spectrosc. 28 (1974), 219. 
[ 30] Escard, J., Brion, D., Bonnet, J. B., Winter, C., Bull. Soc. Chim. France, No 1-2 (1974) 72.

[31] Escard, J., Brion, D., C. R. Hebd. Sèan. Acad. Sci. 276 (1973) B-945.

[32] Escard, J., Brion, D., J. Am. Ceram. Soc. 58 (1975) 296.

[33] Noel-Tenret, C., Friedmann, R. M., Journées d'étude de l'Ecole Supérieure du Verre, Octobre 1973.

[34] Stephenson, D. A., Binkowski, N. J., J. Non-Crystal. Solids 19 (1975) 87.

[35] Budd, S. M., J. Non-Crystal. Solids 19 (1975) 55.

[36] Rynd, J. P., Rastogi, A. K., Surf. Sci. 48 (1975) 22.

[37] Winter, C., Escard, J., Brion, D., Chatenet, B., DonNET, J. B., Silic. Ind. 9 (1975) 231.

[38] Documents ES 208. 11, AEI Scientific Apparatus Inc.
[39] Siegbahn, K., J. Electron Spectros. 5 (1974) 3.

[40] Liang, K. S., J. Non-Crystal. Solids 18 (1975) 197.

[41] Hollinger, G., Kumurdian, P., Mackowski, J. M., Pertosa, P., Porte, L., Tran minh duc, J. Electr. Spectrosc. and Rel. Phen. 5 (1974) 237.

[42] Hollinger, G., Jugnet, Y., Pertosa, P., Tran minh duc, Chem. Phys. Lett. 36 (1975) 441.

[43] Sherchik, N. J., Phys. Rev. Lett. 33 (1974) 1572 ; J. Phys. C : Solid State Phys., 8 (1975) 3767.

[44] Joannopoulos, J. D., Schlüter, M., Cohen, M. L., Phys. Rev. B-11 (1977) 2186.

[45] Robertson, J., Phil. Mag. 34 (1976) 13.

[46] Pantano, C. G. Jr, Dove, D. B., Onoda, G. Y. Jr., J. Non-Crystal. Solids 19 (1975) 55. 